\title{
Application of Affine-Invariant Fourier Descriptors to Recognition of 3-D Objects
}

\author{
KLAUS ARBTER, WESLEY E. SNYDER, SENIOR MEMBER, IEEE, HANS BURKHARDT, \\ AND GERD HIRZINGER
}

\begin{abstract}
In this work, the method of Fourier descriptors has been extended to produce a set of normalized coefficients which are invariant under any affine transformation (translation, rotation, scaling, and shearing). The method is based on a parameterized boundary description which is transformed to the Fourier domain and normalized there to eliminate dependencies on the affine transformation and on the starting point.

Invariance to affine transforms allows considerable robustness when applied to images of objects which rotate in all three dimensions. This is demonstrated by processing silhouettes of aircraft as the aircraft maneuver in three-space.
\end{abstract}

Index Terms-Affine transformation, features, Fourier descriptors, invariants, shape, 3-D parameter estimation, 2-D parameter determination.

\section{INTRODUCTION AND BACKGROUND}

$\mathrm{F}^{\circ}$ OURIER descriptors provide a means for representing the boundary of a two-dimensional shape. The basic idea is: a closed curve may be represented by a periodic function of a continuous parameter, or alternatively, by a set of Fourier coefficients of this function. The coefficients in this collection are referred to as "Fourier descriptors" (FD's). To use these descriptors for pattern classification applications, the curve representation must be normalized with respect to a desired transformation class. If the normalization is exact, it will result in a set of FD's which are invariant with respect to the specified class. Most of the research has been done to date assuming the class of similarity transforms, which allows trans lation and rotation in the plane, and scaling. A free choice of the starting point for the parameterization is assumed as well.

The early similarity-invariant FD's were derived by normalizations performed in the original (spatial) domain completely [4] or partially [23], using the invariant properties of curvature and/or tangent angle. The calculation of these quantities implies the calculation of derivatives, which may be avoided by performing the normalization completely in the Fourier domain. Such Fourier-domain

Manuscript received April 1, 1988; revised August 15, 1989. Recommended for acceptance by J. L. Mundy. This work was supported by the Deutsche Forschungs und Versuchsanstalt für Luft und Raumfahrt.

K. Arbter and G. Hirzinger are with the Deutsche Forschungsanstalt für Luft und Raumfahrt, e.V., Wessling, West Germany.

W. E. Snyder is with the Center for Communications and Signal Processing, North Carolina State University, Raleigh, NC 27695.

H. Burkhardt is with the Technical University of Hamburg, Hamburg, West Germany.

IEEE Log Number 9036113 normalization was initially developed by Granlund [7] and later extended to address the issue of completeness [3], [5].

The class of affine transforms includes the similarity transforms, but in addition allows "shear." Under shear, the shape of the object boundary will no longer be preserved, as illustrated in Fig. 1. Such shape distortion can typically arise if a planar object is observed by a camera under arbitrary orientations with respect to the plane. In this case, the perspective transformation would be the most appropriate, however, the affine transformation may be used as an approximation much closer [20] to the perspective transformation than the similarity transformation would be. Miyatake, Matsuyama, and Nagao [13] proposed an optimizing procedure to produce invariance under affine transformations. An algebraic normalization with respect to affine transforms has recently been published [1]. That paper also contains extensions to the starting point normalization problem as well as algebraic formulae for the Fourier transformation of polygons (see Appendix), similar to those given in Persoon and Fu [17] [16], but extended to discontinuities.

Similarity-invariant FD's have been widely used for recognition of 3-D objects by processing their silhouettes. In the tradition of research [19], [21], [8], [12], [2], [11], [10], we have also chosen to process silhouettes of aircraft. In doing so, the normalized FD's will not be treated as invariant representations, but rather as features for recognition. We applied a small subset of the affine-invariant Fourier descriptors [1] to silhouettes of aircraft which were maneuvering in three-space and compared the resulting clusters of features to those resulting from similarity-invariant FD's of the same data. We found increased robustness of affine-invariant FD's under such 3-D motion.

In this paper, we will cover the theory in sufficient detail to allow the reader to duplicate our results, but will not explore the concepts in depth, nor will we provide proofs herein. For more details, derivations, proofs, and extensions, the reader is referred to Arbter [1].

\section{Data Representation}

In the following discussion, we will consider only boundaries of silhouettes. Therefore, it will be implied, where not stated, that all curves and representation are in the viewing plane of the camera. 


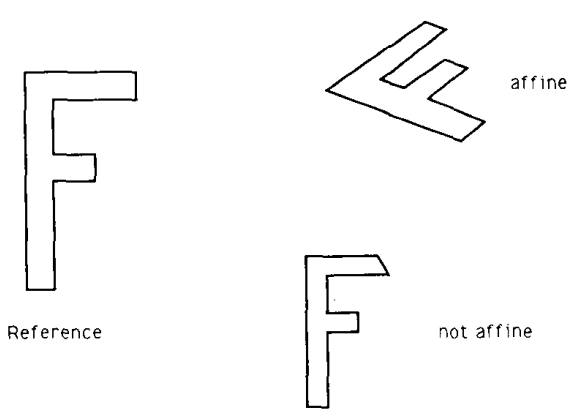

Fig. 1. Affine transformations appear as translation, rotation, and shear.

Any plane curve can be described in the real space $R^{2}$ or in the complex plane $\boldsymbol{C}$.

Using these two representations, an affine transform may be written as

$$
\boldsymbol{x}=A \boldsymbol{x}^{0}+\boldsymbol{b} \quad \operatorname{det}(A) \neq 0
$$

for $x, x^{0} \in \mathbb{R}^{2}, A$ is a $2 \times 2$ matrix, $b$ is a 2 -vector, and $x$ is the affine-transformed version of $x^{0}$ or, using the complex representation,

$$
x=a x^{0}+b x^{0}+c \quad a a^{*}-b b^{*} \neq 0
$$

where $x, x^{0}, a, b, c \in C$, and where $(*)$ represents the complex conjugate operation. In most previous papers on Fourier descriptors, the complex representation has been used, probably because under a similarity transform, (2.2) reduces to $x=a x^{0}+b$, and it is easy to show that $a$ can be eliminated in the Fourier domain by simple normalizations. In our work, we will find it most practical to use the vector representation for the same reason-that it is easier to show the elimination of affine transformation parameters using this notation. Thus, we may say that a contour is an ordered set of points in $R^{2}$, and we will use the term "affine contour" to mean a contour which has undergone an affine transformation.

\section{Parameterization}

The familiar arc length parameterization transforms linearly under any linear transformation up to the similarity transform. Translation and rotation do not affect the arc length, scaling scales the parameter by the same amount, and choice of a starting point introduces only a shift in the parameter. For these reasons, previous authors preferred arc length parameterization. However, the arc length is nonlinearly transformed under an affine transformation. Thus, we introduce a new parameterization and require that such a parameterization have the following two properties:

1) It must be linear under an affine transformation. That is, if $t$ is the parameter used for the observation and $t^{0}$ the parameter used for the reference, we require that for some constant, $c, t=c\left(t^{0}+\tau\right)$. We require this because the Fourier transform preserves the linearity of the affine transformation if and only if the parameter transformation is linear. The Fourier-domain normalization which will be discussed in a later section will compensate for both of these factors.

2) The parameterizing function must yield the same parameterization independent of the initial representation of the contour.

Two candidate parameterizations come to mind which satisfy these criteria: the first is the "affine length" [14]

$$
t=\int_{C} \sqrt[3]{\operatorname{det}\left(\frac{d x}{d \xi}, \frac{d^{2} x}{d \xi^{2}}\right)} d \xi=\int_{C} \sqrt[3]{x_{\xi} y_{\xi \xi}-y_{\xi} x_{\xi \xi}} d \xi
$$

where $x_{\xi}, y_{\xi}$ are the first and $x_{\xi \xi}, y_{\xi \xi}$ are the second derivatives of the components $x(\xi)$ and $y(\xi)$, and $C$ is the path along the curve. We have difficulty with the affine length, since our boundary encoding will eventually be with polygons, and this parameterization involves a second derivative. Use of the second derivative would result in a parameterization which is zero along the sides of the polygon and infinite at the vertices.

Instead, we use a first order form

$$
\begin{aligned}
t & =\frac{1}{2} \int_{c}\left|\operatorname{det}\left(\boldsymbol{x}(\xi), x_{\xi}\right)\right| d \xi \\
& =\frac{1}{2} \int_{c}\left|x(\xi) y_{\xi}-y(\xi) x_{\xi}\right| d \xi .
\end{aligned}
$$

This parameterization will not be invariant for the case $b$ $\neq 0(2.1)$; that is, translation. We avoid this problem by initially moving the coordinate system to the area center, defined by

$$
x_{s}=\frac{2}{3} \frac{\oint_{C} x(\xi) \operatorname{det}\left(x(\xi), x_{\xi}\right) d \xi}{\oint_{C} \operatorname{det}\left(x(\xi), x_{\xi}\right) d \xi}
$$

The area center of an affine contour is the affine transform of the area center. This results from the fact that the affine transformation transforms areas with a constant scale $\operatorname{det}(A)$.

Theorem 1: The parameterization given in (3.2) is linear under an affine transform if $\boldsymbol{b}=0$.

The parameterization of (3.2) may be interpreted geometrically as an area, as shown in Fig. 2. Because of the above-mentioned property of the affine transformation, we find that for a differential area $d F$,

$$
d F=\operatorname{det}(A) d F^{0}
$$

is true, determining a proportional relationship between the parameter differentials of the two affine-related contours.

We note that this parameterization allows discontinuities. For example, in Fig. 3, an instance is shown in which the coordinates undergo a finite change for zero change in subtended area.

We will be taking the Fourier transform of the parameterized boundary, and this type of discontinuity will have 


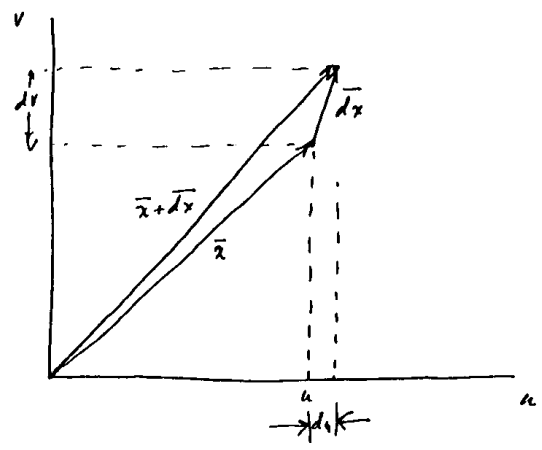

Fig. 2. The enclosed area is $\frac{1}{2}\left|\operatorname{det}\left[\begin{array}{ll}u & d u \\ v v\end{array}\right]\right|$.

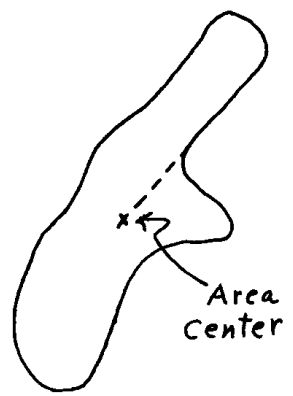

Fig. 3. Rotating counterclockwise about the center of gravity, a discontinuity occurs in the parameterization if the boundary has a finite arc which aligns with the radius.

an effect on our ability to reconstruct the boundary. In particular, a reconstruction of the boundary from the inverse transform will exhibit Gibbs phenomenon (ripples) in the vicinity of the discontinuity [15]. However, the reader should keep in mind that our objective in this work is not image coding or reconstruction, but rather invariant matching, and such phenomena will cause us no problems in matching.

\section{Fourier Transform}

In the usual way, we encode the boundary as a function of the parameter, and take the Fourier transform of the resulting function. Since we are using a vector representation for the boundary, we describe a point on the boundary by a vector function

$$
\boldsymbol{x}=\left[\begin{array}{l}
u(t) \\
v(t)
\end{array}\right]
$$

The Fourier transform is then applied to the functions $u(t)$ and $v(t)$, resulting in a matrix of coefficients

$$
\left[\begin{array}{llll}
\cdots & U_{0} & U_{1} & \\
& V_{0}, & V_{1} &
\end{array}\right] .
$$

Although these coefficients are complex, the functions $u(t)$ and $v(t)$ are real. Therefore, we have

$$
U_{-k}=U_{k}^{*} \quad V_{-k}=V_{k}^{*}
$$

(where $x^{*}$ represents the complex conjugate of $x$ ) and we can discard all coefficients $\left[U_{k}, V_{k}\right]^{\mathrm{T}}$ for $k<0$.

\section{CONSTRUCTION OF INVARIANTS FROM Fourier COEFFICIENTS}

Our image matching problem now has seven parameters. Six come from the elements of $\boldsymbol{A}$ and $\boldsymbol{b}$ (2.1). The seventh is the starting point of the boundary encoding process. From the Fourier coefficients, we need to construct a description of the boundary which is independent of all of these parameters. We will initially ignore the starting point problem, and come back to it in the next section.

We discard the pair $\left[U_{0}, V_{0}\right]^{\mathrm{T}}$ first since it contains no shape information, and second since it and only it depends on translation (shift). The remaining coefficients are shift invariant.

First, we define "relative invariants," that is, a set of numbers $I_{k}, I_{k} \in C$ which satisfy the following relations. Let $I_{k}^{0}$ represent the $k$ th invariant measured on the reference image, and let $I_{k}$ represent the same invariant measured on the observed image. If $I_{k}$ is indeed a relative invariant, it will satisfy

$$
I_{k}=\mu I_{k}^{0} .
$$

Furthermore, $\mu$ will be the same constant for all $k$.

We can easily find a large set of such invariants as follows: Let $\boldsymbol{X}_{k}$ represent the $k$ th Fourier coefficient vector resulting from the transform of the observation. Similarly, let $\boldsymbol{X}_{k}^{0}$ represent the same coefficient from the transform of the reference. Now, if the observation did in fact result from the affine transform $A$ applied to the reference, we have

$$
\boldsymbol{X}_{k}=A \boldsymbol{X}_{k}^{0} \quad k \neq 0
$$

since the Fourier transform is a linear operator. Choose any two coefficients, say $k$, and $p$, and construct the $2 \times$ 2 matrix

$$
\left[\boldsymbol{X}_{k}, \boldsymbol{X}_{p}\right]
$$

Using such a matrix, we may write

$$
\left[\boldsymbol{X}_{k}, \boldsymbol{X}_{p}\right]=A\left[\boldsymbol{X}_{k}^{0}, \boldsymbol{X}_{p}^{0}\right] \text {. }
$$

Taking determinants of both sides, we have

$$
\operatorname{det}\left[\boldsymbol{X}_{k}, \boldsymbol{X}_{p}\right]=\operatorname{det}(A) \operatorname{det}\left[\boldsymbol{X}_{k}^{0}, \boldsymbol{X}_{p}^{0}\right]
$$

and we have invariant scalars which obey the definition of (5.1), where $\mu=\operatorname{det}(A)$. In fact, since we are free to use either the coefficient or its complex conjugate in (5.5), we have $16 \mathrm{~m}^{2}$ invariants, for $m$ coefficients.

To reduce the cardinality of this set (and also its redundancy), we fix $p$ to some constant value, such that

$$
p \neq 0 \text { and } X_{p} \neq 0
$$

and define the set of relative invariants $\Delta_{k}$

$$
\Delta_{k}=\operatorname{det}\left[\boldsymbol{X}_{k}, \boldsymbol{X}_{p}^{*}\right] \text {. }
$$

Theorem 2: The set of relative invariants given in (5.7) is complete and minimal. 
Here, we use the term "complete" to mean: two planar closed curves will have the same set of descriptors if and only if they are affine.

We now derive absolute invariants from the relative invariants of (5.7), eliminating the effects of $\mu$, by simply dividing all the invariants by $\Delta_{p}$

$$
Q_{k}=\frac{\Delta_{k}}{\Delta_{p}}=\frac{\left|\boldsymbol{X}_{k}, \boldsymbol{X}_{p}^{*}\right|}{\left|\boldsymbol{X}_{p}, \boldsymbol{X}_{p}^{*}\right|}=\frac{U_{k} V_{p}^{*}-V_{k} U_{p}^{*}}{U_{p} V_{p}^{*}-V_{p} U_{p}^{*}}
$$

By using the complex conjugate, we get a 1 for the $p$ th invariant and a 0 for the $-p$ th invariant.

In the absence of noise, any $p$ under the restrictions of (5.6) may be chosen. However, in the presence of noise, the signal-to-noise ratio should be as high as possible. Therefore, considering (5.8), one should choose a $p$ for which $\left|\boldsymbol{X}_{p}, \boldsymbol{X}_{p}^{*}\right|$ is as large as possible.

\section{Invariance to Starting Point}

Recalling that $x^{0}\left(t^{0}\right)=\left[u^{0}\left(t^{0}\right), v^{0}\left(t^{0}\right)\right]^{\mathrm{T}}$, in the reference image is related to $x(t)=[u(t), v(t)]^{\mathrm{T}}$ in the observation by

$$
x(t)=A x^{0}\left(t^{0}\right),
$$

we now consider that the two parameterizations, $t$ and $t^{0}$, are not necessarily identical, but may differ by a scale factor and a shift. Therefore, from elementary Fourier transform theory, we have that the $k$ th coefficient of the observation is related to the $k$ th coefficient of the reference by

$$
\boldsymbol{X}_{k}=z^{k} A \boldsymbol{X}_{k}^{0}
$$

where

$$
z=e^{-j 2 \pi \tau / T^{0}}
$$

expresses the parameter shift $\tau$ in the Fourier domain. $T^{0}$ is the parameter period of the reference image.

We now wish to normalize the sequence of $Q_{k}$ in such a way as to eliminate the starting point dependency $z$. We do this by phase normalization. We define phase terms

$$
\Psi_{k_{i}}=\frac{Q_{k_{i}}}{\left|Q_{k_{i}}\right|}
$$

for a subset $\left\{Q_{k_{1}}, Q_{k_{2}}, \cdots, Q_{k_{n}}\right\}$ where the $Q_{k_{i}}$ all have magnitudes different from zero. The sequence

$$
\begin{aligned}
I_{k}=\left|Q_{k}\right| \Psi_{k}^{\rho_{0}} \prod_{i=1}^{n} \Psi_{k_{i}}^{(k-p) \lambda_{i},} & \\
& k= \pm 1, \pm 2, \cdots, \pm \infty
\end{aligned}
$$

is $z$-invariant [1]; where $\rho_{0}$ is the greatest common divisor of the set $\left\{k_{1}-p, k_{2}-p, \cdots, k_{n}-p\right\}$ and where the $\lambda_{i}$ are integer solutions of the diophantine equation

$$
\rho_{0}+\sum_{i=1}^{n} \lambda_{i}\left(k_{i}-p\right)=0 \quad \lambda_{i} \in Z_{0}
$$

This normalization preserves the magnitudes of the $Q_{k}$. The product term may be interpreted as a frequency-linear phase shift (allpass), which does not affect the signal contained in the $Q_{k}$. The phases of the $Q_{k}$ will be multiplied by $\rho_{0}$. Therefore the phase information is reduced by the factor $\rho_{0}$, and unchanged if $\rho_{0}=1$. In this last case, the invariants are also complete. The value of $\rho_{0}$ depends on the index set $k_{1}, k_{2}, \cdots, k_{n}$ which have been chosen for performing the normalization.

For the orders 0 and 1 , we get the $z$-invariants:

$$
\begin{aligned}
& n=0: I_{k}=\left|Q_{k}\right| \\
& n=1: I_{k}=\left|Q_{k}\right| \Psi_{k}^{q-p} \Psi_{q}^{p-k}
\end{aligned}
$$

where the first term eliminates all the phase information and the second preserves all the phase information provided the fixed indexes $q$ and $p$ differ only by one.

The choice of an index set $\left\{k_{1}, k_{2}, \cdots, k_{n}\right\}$ has different aspects. In the presence of noise, one should choose indexes for which the corresponding $Q_{k_{i}}$ have large magnitudes. For general completeness, the index set must lead to $\rho_{0}=1$. For completeness relative to a particular application, one usually uses an order no higher than necessary. In the experiments presented below, we used invariants of order zero.

\section{Application to 3-D Motion}

In the tradition of research [2], [8], [12], [11], [10], [19], [21], [22] in this field, we have also chosen to process silhouettes of aircraft. We emphasize that our objective in this work is object recognition, and not boundary coding or reconstruction, and the experiments are biased toward that objective.

An orthographic projection of a 3-D motion of a planar object can be exactly described by an affine transform. Therefore, in these experiments, our hypothesis was as follows: An orthographic projection of an object which is almost planar (e.g., an airplane), as it undergoes rigid body motions in three dimensions will be very close to an affine transform of some reference view of the object, as long as the motions are not so large as to cause major occlusions. Therefore, if we can describe the shape of the object using features which are affine-invariant, we should have considerable robustness in a recognizer. The experiments have borne out this hypothesis.

In order to ensure that the experiments were well controlled, we synthesized the aircraft images using a quadric surface synthesis program, Qsyn [9]. Qsyn allows the user to specify an object constructed from the union of a number of quadric surfaces, where each surface is defined by its ten quadric coefficients, constraints, and various threespace rigid body motions. The three aircraft illustrated in Fig. 4 were synthesized using an ellipsoid for the fuselage, planes for the wings and tail, and cylinders for the engine. In order to ensure that our algorithms work using shape and shape alone, all three aircraft had the same fuselage and engines, and all three sets of wings subtended exactly the same area when viewed from above.

Each aircraft was rotated about the $x$ and $y$ axes (pitch and yaw), varying in small uniform increments from 0 to 


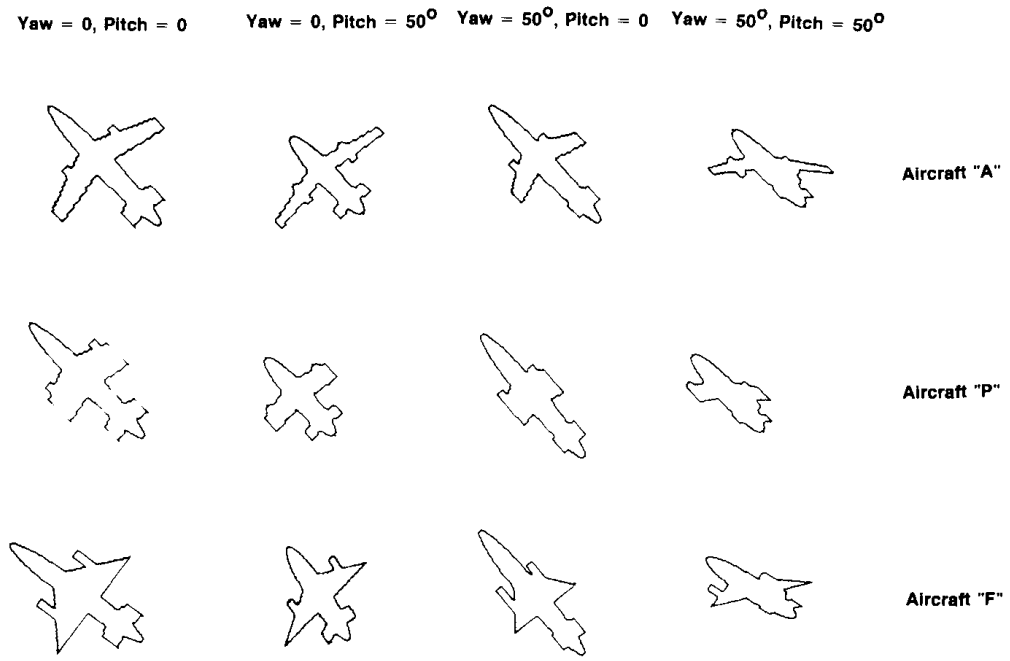

Fig. 4. Example silhouettes of the images used in the experiments

50 degrees. The contour of the resulting image was extracted and the invariants computed. In Fig. 5, a gray scale rendering of one of the images is presented.

\section{A. Clustering of Data}

In Fig. 6, we plot the magnitudes of two of the invariants $\left(I_{-3}\right.$ and $\left.I_{2}\right)$ versus each other, for each of the 50 experiments per aircraft. Boxes represent aircraft $A$, stars represent aircraft $F$, and triangles represent aircraft $P$. The data clearly clusters, using just these two coefficients Therefore, standard statistical pattern recognition techniques will suffice to discriminate between the three classes. The lower portion of Fig. 6 duplicates the experiment illustrated in the upper portion, but using Fourier coefficients normalized in the more traditional manner, resulting in descriptors which are invariant to similarity transforms (rotations and translations in the viewing plane), but not to affine transforms. Although we still observe clustering, the spread of the clusters is significantly larger than in the upper portion of the figure, verifying that the affine-invariant descriptors provide more robust descriptors.

\section{B. Extent of Clustering}

In an attempt to quantify this comparative robustness, the following analysis of the data was undertaken. Five descriptors were chosen, $I_{-3}, I_{-2}, I_{2}, I_{3}$, and $I_{4}$ ( $I_{0}$ does not exist and because we chose $p=1, I_{-1}$ and $I_{1}$ have constant values of 0 and 1 , respectively) and organized into a vector $I$. The scatter matrix of $I$ was computed:

$$
S=\sum I I^{\mathrm{T}},
$$

and the eigenvalues of $S$ were found. The eigenvalues of $S, \lambda_{1}, \cdots, \lambda_{5}$, are monotonically related to the length of the major axes of the five-dimensional hyperellipsoid which describes the distribution of the data. The magnitudes of the $\lambda$ 's thus measure the spread of the invariants.

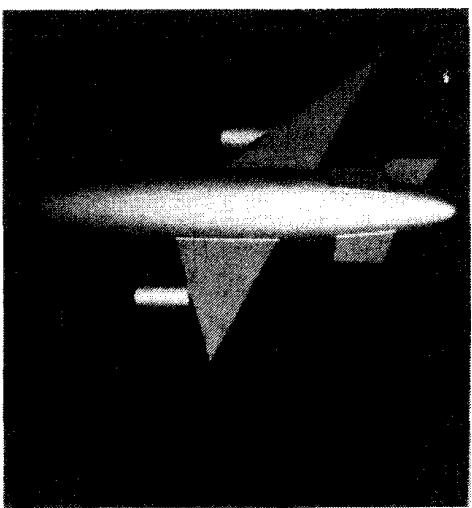

Fig. 5. One pose of aircraft $F$

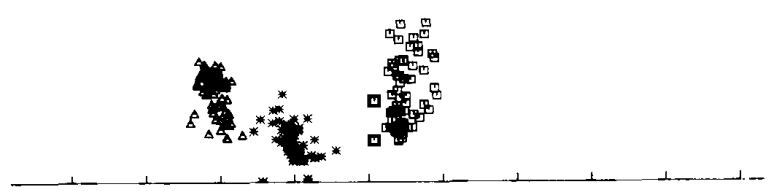

(a)

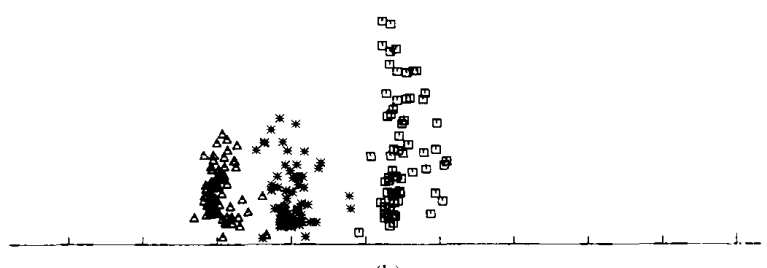

(b)

Fig. 6. Distribution of sample points as the three aircraft of Fig. 4 are rotated in small increments from 0 to 50 degrees. (a) Affine-invariant descriptors. (b) Similarity-invariant descriptors.

(These results are summarized in Table I.) Consistently, the eigenvalues corresponding to the affine-invariants are smaller than the corresponding eigenvalues for the simi- 
TABLE I

Eigenvalues of Scatter Matrix of Five Descriptors for Various Observations of Aircraft (a) $A$, (b) $F$, (c) $P$, Comparing AfFine-InVariant Descriptors With Similarity-Invariant

\begin{tabular}{|c|c|c|c|c|c|}
\hline \multicolumn{2}{|c|}{ Image $A$ Eigenvalues } & \multicolumn{2}{|c|}{ Image $F$ Eigenvalues } & \multicolumn{2}{|c|}{ Image $P$ Eigenvalues } \\
\hline Affine & Similarity & Affine & Similarity & Affine & Similarity \\
\hline 5.666224 & 6.262485 & 2.887337 & 3.557270 & 1.885456 & 2.461978 \\
\hline 0.058298 & 0.196582 & 0.017920 & 0.086499 & 0.019434 & 0.055955 \\
\hline 0.021136 & 0.129334 & 0.006078 & 0.034761 & 0.003039 & 0.004550 \\
\hline 0.005054 & 0.015014 & 0.001871 & 0.012243 & 0.001315 & 0.002261 \\
\hline 0.001792 & 0.000908 & 0.000981 & 0.000700 & 0.000342 & 0.001832 \\
\hline \multicolumn{2}{|c|}{ (a) } & \multicolumn{2}{|c|}{ (b) } & \multicolumn{2}{|c|}{ (c) } \\
\hline
\end{tabular}

larity invariants, and therefore represent more densely packed clusters.

\section{Optimization of Clustering}

Fig. 6 compares the clustering results using only two of the invariants. Since any number of invariants may be considered, one may easily conceive of performing cluster analysis in higher dimensionality spaces, with the hope of increasing the discriminability. To test this capability, the following experiment was performed. The same five invariants as used in Section VII-B were chosen as features to be clustered. A projection of this data from the five-dimensional feature space onto a two-dimensional space was computed and the clustering observed. The projection used was one which optimized the normalized between-cluster distance of the projected data. Specifically, one may define

$$
J=\frac{\left|W^{\mathbf{T}} S_{B} W\right|}{\left|W^{\mathbf{T}} S_{w} W\right|}
$$

as a criterion function to be maximized, where $W$ is the unknown projection matrix; $S_{B}$ is the between-class scatter, which measures the expected distance between the cluster means; and $S_{W}$ is the within-class scatter, which measures the expected variance from the particular cluster means to the individual data points in each cluster. Finding the matrix $W$ which maximized $J$ may be reduced to an eigenvalue problem [18, pp. 118-120].

This projection method was applied to the affine-invariants computed for the three aircraft, and the results were plotted. Those results are not included here because the resulting clustering was slightly worse than that shown in Fig. 6. Upon reflection, the reasons for this inferior performance are quite clear: first, most of the information is contained in the two non-trivial invariants with the lowest-indices, as is often the case in image analysis-most of the picture information is in the low-frequency terms. Second, the criterion function given in (7.1) will tend to find a projection which results in spherical clusters. Since the data is not spherical in five-space, forcing a spherical projection results in a 2-D distribution of points which is not representative.

It is important to keep in mind that the fundamental assumptions underlying both the affine-invariant and the similarity-invariant descriptors have been violated. The observed images are neither affine nor similarity transforms of some reference. Had this been true, the distributions of Fig. 6 would be reduced to single points. (In fact, we have performed this experiment by rotating planar images of printed characters out of the viewing plane, and the distributions of the affine-invariant come very close to single points, even with real, noisy data.) In this experiment, we have shown that rotation of a 3-D object out of the viewing plane is closer to being an affine transformation of its original image than to being a similarity transform, and therefore affine-invariance provides considerable robustness. It is, in fact, because the various aspects of the same object do not produce exact affine images that we chose to use the least sensitive invariants: $I_{k}$ $=\left|Q_{k}\right|$.

It is instructive to compare this work with others which perform similar recognition tasks. Mitchell [12] uses a library of 143 views to accommodate rotation out of the viewing plane. Thus 143 comparisons must be made for each reference. Of course, the 143 models Mitchell uses accommodates a full $90^{\circ}$ of rotation in both pitch and yaw, while the work reported herein allows only $50^{\circ}$. However, only one model is used for that entire $50^{\circ}$ !

\section{FUTURE WORK}

In this work, we have summarized the work of Arbter [1] and applied that work to three-dimensional applications. The summary has shown that it is possible to construct a set of Fourier descriptors which are invariant to arbitrary affine transforms. Experimentally, we have shown that affine invariance is a property which is useful in applications in which image distortions occur which are nearly affine. Three-dimensional motion is one very practical such application.

Since the vector form for the coefficients could easily be extended to more than two dimensions, the method may be expanded to higher dimensional affine-invariant curve descriptions. For the 3-D-case, we have the correspondence:

$$
\begin{aligned}
u(t) & \leftrightarrow U(k) \\
v(t) & \leftrightarrow V(k) \\
w(t) & \leftrightarrow W(k)
\end{aligned}
$$


and must, therefore, deal with $3 \times 3$ matrices

$$
\left[\begin{array}{ccc}
U(k) & U(q) & U(r) \\
V(k) & V(q) & V(r) \\
W(k) & W(q) & W(r)
\end{array}\right]
$$

instead of the $2 \times 2$ matrices as above. However, as far as closed curves are concerned, we have the same basic concept. It would be interesting to explore the possibilities of further extensions, such as to surfaces rather than curves [6], or to partial shapes [12].

\section{APPENDIX}

Calculating the Fourier Descriptors of Polygons

Let $u_{i}, v_{i}: i=0, \cdots, N-1$ be the coordinates of the $N$ vertices of an oriented polygon in $\mathbb{R}^{2}$, and $t_{i}$ their parameter values. Furthermore $\left(u_{N}, v_{n}\right)=\left(u_{0}, v_{0}\right)$ and $t_{N}$ $=t_{0}+T$. The Fourier transformation is given by

$$
\begin{aligned}
\left(\begin{array}{c}
U_{k} \\
V_{k}
\end{array}\right)= & \frac{T}{(2 \pi k)^{2}} \sum_{i=0}^{N-1} \frac{1}{t_{i+1}-t_{i}}\left(\begin{array}{c}
u_{i+1}-u_{i} \\
v_{i+1}-u_{i}
\end{array}\right) \\
& \cdot\left(\Phi_{k, i+1}-\Phi_{k, i}\right)\left[1-\delta\left(t_{i+1}-t_{i}\right)\right] \\
& +\frac{j}{2 \pi k} \sum_{i=0}^{N-1}\left(\begin{array}{c}
u_{i+1}-u_{i} \\
v_{i+1}-u_{i}
\end{array}\right) \Phi_{k, i} \delta\left(t_{i+1}-t_{i}\right),
\end{aligned}
$$

where

$$
\Phi_{k, i}=\exp \left\{-j 2 \pi k t_{i} / T\right\}
$$

and

$$
\delta\left(t_{i+1}-t_{i}\right)= \begin{cases}1 & \text { if } t_{i+1}=t_{i} \\ 0 & \text { if } t_{i+1} \neq t_{i}\end{cases}
$$

In contrast to the FFT this transformation takes the indefinite set of all the points on the polygon into account, does not need constant parameter intervals and allows the transformation of discontinuous functions.

The area parameterization for polygons is given by

$$
\begin{array}{r}
t_{0}=0 ; t_{i+1}=t_{i}+\frac{1}{2}\left|\left(u_{i}^{\prime} v_{i+1}^{\prime}-u_{i+1}^{\prime} v_{i}^{\prime}\right)\right|, \\
i=0,1,2, \cdots, N-1 ; T=t_{N},
\end{array}
$$

with

$$
\begin{aligned}
\left(\begin{array}{l}
u_{i}^{\prime} \\
v_{i}^{\prime}
\end{array}\right) & =\left(\begin{array}{c}
u_{i}-u_{A C} \\
v_{i}-v_{A C}
\end{array}\right) \\
\left(\begin{array}{l}
u_{A C} \\
v_{A C}
\end{array}\right) & =\frac{\sum_{i=0}^{N-1}\left(u_{i} v_{i+1}-u_{i+1} v_{i}\right)\left(\begin{array}{l}
u_{i}+u_{i+1} \\
v_{i}+v_{i+1}
\end{array}\right)}{3 \sum_{i=0}^{N-1}\left(u_{i} v_{i+1}-u_{i+1} v_{i}\right)} .
\end{aligned}
$$

\section{REFERENCES}

[1] K. Arbter, "Affine-invariant Fourier descriptors," in From Pixels to Features. Amsterdam, The Netherlands: Elsevier Science, 1989.
[2] K. Arbter and H. Burkhardt, "Ein beitrag zur anwendung von Fourierdeskriptoren für die lage- und grösseninvarieante objekterkennung," Zeitschrift für Flugwissenschaften und Weltraumforschung, vol. 8, no. 1, 1984 .

[3] H. Burkhardt, Transformationen zur lageinvarianten Merkmalgewinnung. Dusseldorf, West Germany: VDI-Verlag, 1979, FortschrittBerichte der VDI-Zeitschriften Reihe 10, no. 7

[4] R. L. Cosgriff, "Identification of shape," Ohio State Univ. Res. Foundation, Columbus, OH, Tech. Rep. ASTIA AD 254 792, Dec. 1960.

[5] T. Crimmins, "A complete set of Fourier descriptors for two-dimensional shapes," IEEE Trans. Syst., Man, Cybern., vol. SMC-12, no, $6,1982$.

[6] G. Eichmann and M. Jankowski, "Fourier shape descriptors for surfaces of multidimensional closed volumes," Proc. SPIE, pp. $482-$ 487.

[7] G. H. Granlund, "Fourier preprocessing for hand printed character recognition," IEEE Trans. Comput., vol. C-21, Feb. 1972.

[8] F. P. Kuhl and C. R. Giardina, "Elliptic Fourier features of a closed contour," Comput. Graphics Image Processing, vol. 18, 1982.

[9] M. Lanzo, "Qsyn, quadric surface synthesizer," Communication Unlimited, Raleigh, NC, Tech. Rep. 87-1, 1987.

[10] C. C. Lin and R. Chellappa, "Classification of partial 2-D shapes using Fourier descriptors," in Proc. IEEE Conf. Computer Vision and Pattern Recognition, Miami Beach, FL, June 1986.

[11] J. Ma, C. Wu, and X. Lu, "A fast shape descriptor," Comput. Vision Graphics, Image Processing, vol. 34, pp. 282-291, 1986.

[12] O. R. Mitchell and T. A. Grogan, "Global and partial shape discrimination for computer vision," Opt. Eng., vol. 23, no. 5, 1984.

[13] T. Miyatake, T. Matsuyama, and M. Nagao, "Affine transform invariant curve recognition using Fourier descriptors (original in Japanese)," Trans. Inform. Processing Soc. Japan, vol. 24, no. 1, pp. $64-71,1983$.

[14] J. Naas and H. Schmid, Mathematisches Wörterbuch. Berlin: Academie-Verlag, 1961.

[15] A. Papoulis, The Fourier Integral and Its Applications. New York: McGraw-Hill, 1962.

[16] E. Persoon and K. S. Fu, "Shape discrimination using Fourier descriptors," IEEE Trans. Syst. Man., Cybern., vol. SMC-7, no. 3, Mar. 1977.

[17] _ " "Shape discrimination using Fourier descriptors," in Proc. 2nd IJCPR, Aug. 1974.

[18] R. R. Duda and P. Hart, Pattern Classification and Scene Analysis. New York: Wiley, 1973

[19] C. W. Richard and H. Hemami, "Identification of three-dimensional objects using Fourier descriptors of the boundary curve,"' IEEE Trans. Syst., Man., Cybern., vol. SMC-4, no. 4, July 1974.

[20] D. W. Thompson and J. L. Mundy, "Three dimensional model matching from an unconstrained viewpoint," in Proc. Conf. Rabotics and Automation, Raleigh, NC, 1987.

[21] T. P. Wallace and O. R. Mitchell, "Analysis of three-dimensional movement using Fourier descriptors," IEEE Trans. Pattern Anal. Machine Intell., vol. PAMI-2, no. 6, 1980.

[22] T. P. Wallace and P. A. Wintz, "An efficient three-dimensional aircraft recognition algorithm using normalized Fourier descriptors,' Comput. Graphics Image Processing, vol. 13, pp. 99-126, 1980.

[23] C. Zahn and R. Roskies, "Fourier descriptors for plane closed curves," IEEE Trans. Comput., vol. C-21, no. 3, Mar. 1972.

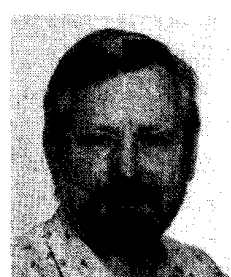

Klaus Arbter received the Ing. (grad.) degree in precision mechanics from the School of Engineering of Aalen, West Germany, and the Dipl.-Ing. in electrical engineering from the Technical University of Munich, West Germany.

He was employed by the Siemens Company, Munich, during 1973-1976 and joined the German Aerospace Research Establishment (DLR), Oberpfaffenhofen, in 1976, where he is currently a Research Scientist in the Robotics Research Laboratory. He worked on airborne navigation system analysis, digital radar signal simulation, and robot motion programming. His main interest is pattern recognition. 


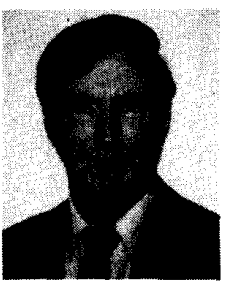

Wesley E. Snyder (S'71-M'75-SM'80) received the B.S. degree in electrical engineering from North Carolina State University, in 1968, and the M.S. and $\mathrm{Ph} . \mathrm{D}$. degrees from the University of Illinois, Urbana, in 1973 and 1975, respectively. Upon completion of his work in Illinois, he returned to North Carolina where he is a member of the faculty of the Department of Electrical and Computer Engineering. He is the author of 48 refereed publications.

Dr. Snyder has participated in many IEEE activities, including the Robotics and Automation Council (member, 19821989), and the Neural Networks Council (member, 1988-1990). He has participated in the organization of several major conferences, including being local arrangements chair of the 1987 International Conference on Robotics and Automation, and the General Chair of the 1989 International Joint Conference on Neural Networks. In addition, he has been a session chair and/or on the program committee of many other conferences.

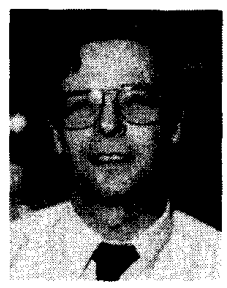

Hans Burkhardt was born in Germany in 1944 He received the Dipl.-Ing. degree in electrical engineering in 1969, the Dr.-Ing. degree in 1974, and the Venia Legendi in 1979 from the University of Karlsruhe, Germany.

He joined the Institut für Mess- und Regelungstechnik at the University of Karlsruhe as a Research Assistant in 1969 and became a Lecturer in 1975. During 1980-1981 he had a fellowship at the IBM Research Laboratory, San Jose, CA. In 1981 he became Professor for control and sig- nal theory at the University of Karlsruhe. In 1985 he became full Professor at the Technical University of Hamburg, heading since then an Institute in the Department of Computer Science. Since 1975 his main research interest has been in digital image processing and pattern recognition. He has published over 50 papers and given more than 70 lectures. He is a consultant of the German Science Foundation (DFG) and of several international journals.

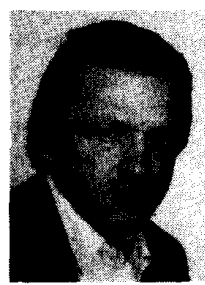

Gerd Hirzinger received the Dipl.-Ing. and Doctor's degrees from the Technical University of Munich, West Germany, in 1969 and 1974, respectively.

In 1969 he joined the German Aerospace Research Establishment (DLR) where he first worked on fast digital control systems. Since 1976 he has been head of the Robotics Research Laboratory of DLR and has published numerous papers on robot sensing and sensory feedback.

Dr. Hirzinger is a member of the Administrative Committee (AdCom) of the IEEE Robotics and Automation Society and is Chairman of the German Council on Robot Control. 